\title{
Decreased levels of metabolic enzymes in pancreatic islets of patients with type 2 diabetes
}

\author{
M. J. MacDonald • M. J. Longacre • E.-C. Langberg • \\ A. Tibell • M. A. Kendrick • T. Fukao • C.-G. Ostenson
}

Received: 21 November 2008 / Accepted: 29 January 2009 /Published online: 19 March 2009

(C) Springer-Verlag 2009

\begin{abstract}
Aims/hypothesis Glucose-stimulated insulin secretion is defective in patients with type 2 diabetes. We sought to acquire new information about enzymes of glucose metabolism, with an emphasis on mitochondrial enzymes, by comparing pancreatic islets of type 2 diabetes patients with those of non-diabetic controls.

Methods Expression of genes encoding 13 metabolic enzymes was estimated with microarrays and activities of up to nine metabolic enzymes were measured.

Results The activities of the mitochondrial enzymes, glycerol phosphate dehydrogenase, pyruvate carboxylase (PC) and succinyl-CoA:3-ketoacid-CoA transferase
\end{abstract}

Electronic supplementary material The online version of this article (doi:10.1007/s00125-009-1319-6) contains supplementary material, which is available to authorised users.

M. J. MacDonald $(\bowtie) \cdot$ M. J. Longacre $\cdot$ M. A. Kendrick Children's Diabetes Center, University of Wisconsin

School of Medicine and Public Health,

1300 University Avenue,

Madison, WI 53706, USA

e-mail: mjmacdon@wisc.edu

E.-C. Langberg $\cdot$ C.-G. Ostenson

Department of Molecular Medicine and Surgery,

Karolinska Institutet,

Stockholm, Sweden

\author{
A. Tibell \\ Department of Transplantation Surgery, Karolinska Institutet, \\ Stockholm, Sweden \\ T. Fukao \\ Medical Information Sciences Division University Graduate \\ School and Department of Pediatrics, \\ Gifu University, \\ Gifu, Japan
}

(SCOT) were decreased by $73 \%, 65 \%$ and $92 \%$, respectively, in the diabetic compared with the non-diabetic islets. ATP citrate lyase, a cytosolic enzyme of the mitochondrial citrate pyruvate shuttle, was decreased $57 \%$. Activities of propionyl-CoA carboxylase, NADP-isocitrate dehydrogenase, cytosolic malic enzyme, aspartate aminotransferase and malate dehydrogenase were not significantly different from those of the control. The low activities of PC and SCOT were confirmed with western blots, which showed that their protein levels were low. The correlation of relative mRNA signals with enzyme activities was good in four instances, moderate in four instances and poor in one instance. In diabetic islets, the mRNA signal of the islet cell-enriched transcription factor musculoaponeurotic fibrosarcoma oncogene homologue $\mathrm{A}$, which regulates expression of islet genes, including the $P C$ gene, was decreased to $54 \%$ of the control level. $P C$ activity and protein levels in the non-diabetic islets were significantly lower than in islets from non-diabetic rodents.

Conclusions/interpretation Low levels of certain islet metabolic enzymes, especially mitochondrial enzymes, are associated with human type 2 diabetes.

Keywords ATP citrate lyase $\cdot$ Mitochondrial glycerol phosphate dehydrogenase $\cdot$ Pyruvate carboxylase $\cdot$ SuccinylCoA:3-ketoacid-CoA transferase
Abbreviations
ATPCL ATP citrate lyase
IDH Isocitrate dehydrogenase
mGPD Mitochondrial glycerol phosphate dehydrogenase
PC Pyruvate carboxylase
PCC Propionyl-CoA carboxylase
SCOT Succinyl-CoA:3-ketoacid-CoA transferase 


\section{Introduction}

Much of our understanding of the beta cell in type 2 diabetes comes from studies of rodent islets and clonal beta cell lines. Because it remains uncertain how closely these models reflect the pathophysiology of the human beta cell, new basic information is needed to formulate new hypotheses about defective insulin secretion in human diabetes. Mitochondrial metabolite shuttles and biosynthetic processes, such as anaplerosis, are thought to be involved in glucose metabolism, which signals or supports insulin secretion. Studies of pancreatic islets isolated from rodent models of type 2 diabetes have shown decreases in certain mitochondrial enzymes, such as mitochondrial glycerol phosphate dehydrogenase (mGPD), the key enzyme of the glycerol phosphate shuttle, and pyruvate carboxylase (PC), an anaplerotic enzyme
[1-4]. ATP citrate lyase (ATPCL), a cytosolic enzyme of the mitochondrial citrate pyruvate shuttle, is also believed to play a role in the conversion of citrate, an important product of anaplerosis, into precursors for lipid synthesis and signalling in the beta cell. ATPCL is plentiful in islets of rodents $[5,6]$. However, its level in human islets is unknown. Except for one report of low mGPD in islets of patients with type 2 diabetes [7], the levels of metabolic enzymes in islets in diabetic humans have not been reported.

In the current study we had the uncommon opportunity to study pancreatic islets isolated from up to five deceased human donors with type 2 diabetes. Recent reports of the function of islets from diabetic donors have not included measurements of enzymes of intermediary metabolism. We attempted to obtain new information about metabolic pathways in islets in human diabetes by estimating the

Table 1 Low gene transcription and/or enzyme activities of mGPD, PC, ATPCL and SCOT in pancreatic islets from humans with type 2 diabetes compared with those from non-diabetic controls

\begin{tabular}{|c|c|c|c|c|c|c|c|}
\hline \multirow[t]{2}{*}{ Enzyme } & \multicolumn{4}{|c|}{ Gene expression ( $\%$ of non-diabetic control) } & \multicolumn{3}{|c|}{ Islets enzyme activity $\left(\mathrm{nmol} \mathrm{min}^{-1}\left[\mathrm{mg}\right.\right.$ homogenate protein $\left.\left.{ }^{-1}\right]\right)$} \\
\hline & \multicolumn{3}{|c|}{ Individual values } & \multirow{2}{*}{$\begin{array}{l}\text { Average values } \\
58 \pm 7(3)^{\mathrm{c}}\end{array}$} & \multirow{2}{*}{$\frac{\text { Non-diabetic control }}{23 \pm 2.5(4)}$} & \multirow{2}{*}{$\frac{\mathrm{T} 2 \mathrm{DM}}{6.2 \pm 0.7(4)}$} & \multirow{2}{*}{$\frac{\mathrm{T} 2 \mathrm{DM} \text { relative to control }(\%)}{27^{\mathrm{a}}}$} \\
\hline mGPD & 74 & 45 & 54 & & & & \\
\hline $\mathrm{PC}^{\mathrm{e}}$ & 85 & 82 & & 84 & $2.3 \pm 0.2(4)$ & $0.8 \pm 0.06(5)$ & $35^{\mathrm{a}}$ \\
\hline $\mathrm{PCC}$ & & & & & $15 \pm 2(4)$ & $13 \pm 3(5)$ & $87^{\mathrm{d}}$ \\
\hline $\operatorname{PCC} \alpha$ & 90 & 40 & 61 & $64 \pm 12(3)^{\mathrm{d}}$ & & & \\
\hline PCC $\beta$ & 71 & 45 & 63 & $60 \pm 6(3)^{\mathrm{b}}$ & & & \\
\hline ATPCL & 43 & 61 & 37 & $47 \pm 6(3)^{b}$ & $28 \pm 4(4)$ & $12 \pm 3(5)$ & $43^{\mathrm{c}}$ \\
\hline SCOT & 40 & 48 & & 44 & $3.8 \pm 0.6(3)$ & $0,0.3$ & 8 \\
\hline \multicolumn{5}{|c|}{ Aspartate aminotransferase } & $505 \pm 54(4)$ & $434 \pm 6(4)$ & $86^{\mathrm{d}}$ \\
\hline Cyto & 59 & 90 & & 75 & & & \\
\hline Mito & 105 & 109 & & 107 & & & \\
\hline \multicolumn{5}{|c|}{ Malate dehydrogenase } & $3,000 \pm 220(4)$ & $3,100 \pm 320(4)$ & $103^{\mathrm{d}}$ \\
\hline Cyto & 63 & 94 & & 79 & & & \\
\hline Mito & 71 & 73 & 40 & $61 \pm 9(3)^{\mathrm{c}}$ & & & \\
\hline \multicolumn{8}{|l|}{ Malic enzyme } \\
\hline Malic enzyme 1 & 90 & 140 & 120 & $117 \pm 12(3)^{\mathrm{d}}$ & $31 \pm 3(4)$ & $31 \pm 4(4)$ & $100^{\mathrm{d}}$ \\
\hline Malic enzyme 2 & 80 & 91 & 110 & $94 \pm 7(3)^{d}$ & & & \\
\hline Malic enzyme 3 & 70 & 90 & 100 & $87 \pm 7(3)^{\mathrm{d}}$ & & & \\
\hline NADP IDH & & & & & $85 \pm 14(4)$ & $147 \pm 29(5)$ & $173^{\mathrm{d}}$ \\
\hline Cyto & 77 & 44 & 54 & $58 \pm 8(3)^{\mathrm{c}}$ & & & \\
\hline Mito & 300 & 340 & & 320 & & & \\
\hline
\end{tabular}

'Gene expression' indicates the signal strength of mRNAs that encode subunits or isoforms of enzymes from islets of a diabetic donor compared with those from a non-diabetic donor probed in the same mRNA microarray chip. Enzyme activities are the total enzyme activity in the homogenate or cytosol of whole cells

Results are expressed as the individual values and as the mean $\pm \mathrm{SE}$ when the number (in parentheses) of samples $\geq 3$. The SEs of control gene expression values (not shown) were $9.8 \%$ or lower in all cases

${ }^{\mathrm{a}} p \leq 0.005,{ }^{\mathrm{b}} p \leq 0.01,{ }^{\mathrm{c}} p \leq 0.05$ and ${ }^{\mathrm{d}}$ not significant, for averages of diabetic islets vs control islets when enough samples available to permit statistical analysis

${ }^{\text {e }}$ Signals of the $P C$ gene were very weak

cyto, cytosolic; mito, mitochondrial; T2DM, type 2 diabetes mellitus 
activities of mGPD, PC, ATPCL and propionyl-CoA carboxylase (PCC) (not lowered in rodent models of diabetes [3, 4]), as well as succinyl-CoA:3-ketoacid-CoA transferase (SCOT), a mitochondrial biosynthetic enzyme, which forms acetoacetate, a carrier of carbon from mitochondria to the cytosol, where it is a precursor for the synthesis of short-chain acyl-CoAs and lipids [6]. We also measured the activities of malate dehydrogenase, malic enzyme, NADP isocitrate dehydrogenase (IDH) and aspartate aminotransferase, which are present in both mitochondrial and cytosolic isoforms in many organs of most genera. In addition, we performed gene expression microarray estimates of the levels of mRNA transcripts that encode these enzymes, including enzymes present as cytosolic and mitochondrial isoforms.

\section{Methods}

Pancreatic islets were isolated from pancreases of five human donors with type 2 diabetes and four non-diabetic donors. The study was approved by the Human Research Ethics Committee of the Karolinska Institutet. Clinical characteristics of the pancreas donors are described in the Electronic supplementary material [ESM] Table 1. For details of the methods for measurements of the maximum velocity $\left(V_{\max }\right)$ of enzyme activities, see ESM. Relative signals of mRNAs encoding the enzymes were estimated with gene expression microarrays with diabetic islet samples paired with non-diabetic islet samples on the same chips as described [8] (ESM).

\section{Results}

Enzyme activities Table 1 shows the activities of nine enzymes in pancreatic islets of the diabetic and non-diabetic control donors. The average activities of mGPD and PC in islets from the diabetic patients were $27 \%$ and $35 \%$, respectively, of the average activities in islets from nondiabetic donors. The average activity of ATPCL in the islets from diabetic patients was $43 \%$ of the control average. Islets from only two diabetic patients were available at the time we became aware of the role of SCOT in insulin secretion [6]. In these two individuals, SCOT activity equalled only $8 \%$ of the control average. To test whether the decrease in SCOT activity was due to denaturation of the SCOT protein during storage, an immunoblot was performed with an anti-SCOT antibody. This confirmed that the low SCOT activity was due to decreased SCOT protein (Fig. 1a). Similarly, the low level of PC in the diabetic islets was confirmed with a Western blot probed with streptavidin, which tightly binds to biotin-containing proteins. The

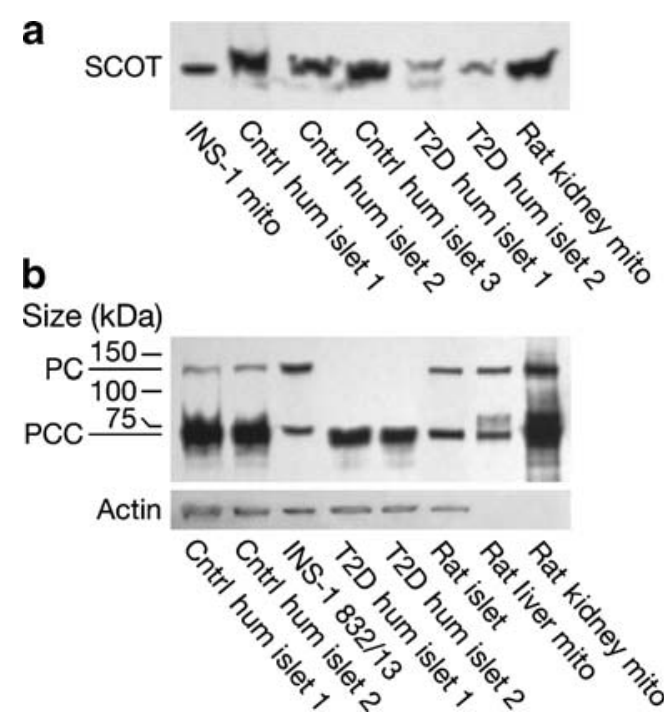

Fig. 1 Decreased SCOT (a) and PC (b) proteins in islets of donors with type 2 diabetes. SCOT and PC in islets of diabetic donors were compared with those in islets of non-diabetic human controls and other cell types as controls. a Immunoblot of SCOT. Protein was quantified as $10,15,6,10,15,15$ and $10 \mu \mathrm{g}$ protein/lane (lanes 1 to 7 , from left to right). b Streptavidin blot. Protein was quantified as $15 \mu \mathrm{g}$ protein/lane in lanes 1 to 6 and $5 \mu \mathrm{g}$ protein/lane in lanes 7 and 8 (from left to right). The actin band shows equal loading of protein across the lanes. T2D, type 2 diabetes. Cntrl, control; mito, mitochondrial; hum, human

western blot also confirmed that PCC, another biotin carboxylase, was not low in the islets of the diabetic patients (Fig. 1b). The average activities of PCC, aspartate aminotransferase, malate dehydrogenase and malic enzyme differed from the average control values by less than $20 \%$. The activities of NADP IDH varied extensively among the islets from the diabetic and control donors and the difference between the average values of the two groups was not statistically significant.

Gene expression The correlation between the decreases in signal strength of mRNAs encoding the various enzymes and the decreases in enzyme activities in diabetic islets relative to non-diabetic islets was good in four cases, moderate in four cases and poor in one case (Table 1). Although gene expression microarray analyses are less quantitative than measurements of enzyme activity, this may nevertheless indicate that the decreases in some cases were caused by decreased gene transcription. It should also be noted that the microarrays measure mRNA signal strength and are less indicative of actual mRNA levels than measurements made with quantitative PCR. The 53\% decrease in the signal of the $A C L Y$ gene encoding ATPCL in the diabetic islets corresponded well with the $57 \%$ decrease in its enzyme activity. The signal of the GPD2 gene encoding mGPD was decreased by $42 \%$, which was 
somewhat less than the decrease in its enzyme activity. There were only small decreases in PC gene signals. However, the signal of $P C$ in the control and diabetic samples was weak. It is noteworthy that the low $P C$ expression correlates with the low level of PC activity (Table 1) and protein (Fig. 1b) in islets from non-diabetic donors compared with the activity [4] and protein (Fig. 1b) in islets of normal rats and the INS-1 cell line. The signals of mRNAs encoding the cytosolic and mitochondrial isoforms of malate dehydrogenase were decreased to a slightly greater degree than the decrease in total activity of malate dehydrogenase. The relatively unchanged signals of mRNAs that encode aspartate aminotransferases and malic enzymes correlated with their normal enzyme activities. The relative signals of the mRNAs that encode the PCC $\alpha$ and $\beta$ subunits were decreased $36 \%$ and $40 \%$, respectively, a somewhat greater decrease than the slight decrease in PCC enzyme activity. The mRNA signal encoding the cytosolic isoform of NADP IDH was decreased $42 \%$, whereas the signal of the mitochondrial isoform of this enzyme was increased threefold. The average enzyme activity reflects the combined activities of both isoforms of NADP IDH. It equalled $173 \%$ of the control values and was close to the average of the mRNA signals. However, this may be coincidental as activities of these enzymes varied widely among the control and diabetic islets.

\section{Discussion}

The current results show that the activities of mGPD and PC were decreased in pancreatic islets of human patients with type 2 diabetes, just as they are in islets of rodent models of diabetes [1-4]. Similarly to rodent islets, where PCC is not decreased in diabetes [3, 4], PCC was not decreased in islets from human donors with type 2 diabetes. The activities of ATPCL and SCOT in islets in diabetes have not been previously reported. ATPCL enzyme activity and its mRNA signal were about 55\% lower in the islets of the human diabetic donors than in those from non-diabetic donors. In islets of the two diabetic patients studied, SCOT activity and protein were decreased $>80 \%$.

The reason for the low levels of MGPD, PC, ATPCL and SCOT in islets in type 2 diabetes is unknown. The activities of mGPD and PC in normal rat and human islets are significantly higher than in most other tissues $[1,4,11]$. Although there is some evidence that beta cell mass is decreased in type 2 diabetes, no more than 20 to $30 \%$ of the decreases in these enzymes in the diabetic islets used in our study can be explained by decreased beta cell mass [8]. Because alleviation of hyperglycaemia with insulin in rodent models of diabetes normalises islet levels of mGPD and PC, it has been suggested that the decreases in these enzymes are a consequence of the diabetes [3]. However, it remains to be fully determined whether: (1) the decreases in any of these enzymes are entirely secondary to diabetes; (2) they themselves in some way contribute to defective insulin secretion in diabetes; or (3) a combination of both mechanisms is in play. In this regard, the lower signals of mRNAs encoding some of the enzymes in the islets of the diabetes patients are consistent with decreased gene transcription. It is significant that the signal of mRNA encoding musculoaponeurotic fibrosarcoma oncogene homolog A, an islet cell-enriched transcription factor that upregulates $P C$ expression [9] and is reduced by glucotoxicity [10], was decreased by $46 \pm 7 \%(n=3)$ in the islets of donors with diabetes compared with control islets. Finally, it is noteworthy that the $P C$ mRNA signal, protein and activity in islets from non-diabetic humans were much lower than in islets from non-diabetic rats (Table 1, Fig. 1b) [4] (M. J. MacDonald, unpublished observations). Because of the central role of $\mathrm{PC}$ in insulin secretion in rodent islets and in clonal cell lines, the role of PC in the human beta cell deserves further investigation.

Acknowledgements This work was supported by NIH Grant DK28348 and the Oscar C. Rennebohm Foundation (to M. J. MacDonald), and by Swedish Research Council and Swedish Diabetes Association (to C. G. Ostenson). We thank M. J. Fallon and M. A. Johnson for excellent technical assistance.

Duality of interest The authors declare that there is no duality of interest associated with this manuscript.

\section{References}

1. Giroix M-H, Rasschaert J, Bailbe D et al (1991) Impairment of glycerol phosphate shuttle in islets from rats with diabetes induced by neonatal streptozocin. Diabetes 40:227-232

2. Ostenson C-G, Abdel-Halim SM, Rasschaert J et al (1993) Deficient activity of FAD-linked glycerophosphate dehydrogenase in islets of GK rats. Diabetologia 36:722-726

3. MacDonald MJ, Efendic S, Ostenson CG (1996) Normalization by insulin treatment of low mitochondrial glycerol phosphate dehydrogenase and pyruvate carboxylase in pancreatic islets of the GK rat. Diabetes 45:886-890

4. MacDonald MJ, Tang J, Polonsky KS (1996) Low mitochondrial glycerol phosphate dehydrogenase and pyruvate carboxylase in pancreatic islets of Zucker diabetic fatty rats. Diabetes 45:16261630

5. Berne C (1975) Nicotinamide adenine dinucleotide phosphateconverting enzymes and adenosine triphosphate citrate lyase in some tissues and organs of New Zealand obese mice with special reference to the enzyme pattern of the pancreatic islets. J Histochem Cytochem 23:660-665

6. MacDonald MJ, Smith AD III, Hasan NM, Sabat G, Fahien LA (2007) Feasibility of pathways for transfer of acyl groups from mitochondria to the cytosol to form short chain acyl-CoAs in the pancreatic beta cell. J Biol Chem 282:30596-30606

7. Fernandez-Alvarez J, Conget I, Rasschaert J, Sener A, Gomis R, Malaisse WJ (1994) Enzymatic, metabolic and secretory patterns 
in human islets of type 2 (non-insulin-dependent) diabetic patients. Diabetologia 37:177-181

8. Ostenson C-G, Gaisano H, Sheu L, Tibell A, Bartfai T (2006) Impaired gene and protein expression of exocytotic soluble $\mathrm{N}$-ethylmaleimide attachment protein receptor complex proteins in pancreatic islets of type 2 diabetic patients. Diabetes 55:435440
9. Wang H, Brun T, Kataoka K, Sharma AJ, Wollheim CB (2007) MAFA controls genes implicated in insulin biosynthesis and secretion. Diabetologia 50:348-358

10. Harmon JS, Stein R, Robertson RP (2005) Oxidative stressmediated, post-translational loss of MafA protein as a contributing mechanism to loss of insulin gene expression in glucotoxic beta cells. J Biol Chem 280:11107-11113 